\title{
A Blockchain-based Service Provider Validation and Verification Framework for Health-care Virtual Organization
}

\author{
Hoger Mahmud ${ }^{1,2}$, Joan $\mathrm{Lu}^{2}$, Qiang $\mathbf{X u}^{3}$ \\ ${ }^{1}$ Department of Computer Science, College of Science and Technology, University of Human Development, Kurdistan Region, \\ Iraq, ${ }^{2}$ Department of Computing Science, School of Computing and Engineering, University of Huddersfield, Huddersfield, UK, \\ ${ }^{3}$ Department of Engineering, School of Computing and Engineering, University of Huddersfield, Huddersfield, UK
}

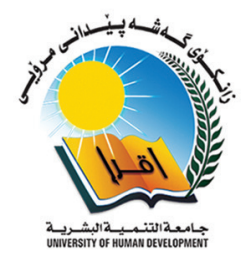

\section{A B S T R A C T}

Virtual organization (VO) and blockchain are two newly emerging technologies that researchers are exploring their potentials to solve many information and communication technology unaddressed problems and challenges. Health care is one of the sectors that are very dynamic, and it is in need of constant improvement in the quest to better the quality of cares and reduce cost. One of the hotlines of research in the sector is the use of information and communication technology to provide health care, and this is where the concept of virtual health care is relevant. In virtual health care, patients and care providers are collaborating in virtual settings where two of the most difficult challenges are verifying and validating the identity of the communicating parties and the information exchanged. In this paper, we propose a conceptual framework using blockchain technology to address the health-care provider and record verification and validation issue. The framework is specific to health-care systems developed based on Virtual Breeding Environment and VO. We outline and explain each step in the the framework and demonstrate its applicability in a simple health-care scenario. This paper contributes toward the continuing effort to address user identity and information verification and validation issues in virtual settings in general and in health care in specific.

Index Terms: Blockchain, Conceptual framework, Validation and verification, Virtual health care, Virtual organization

\section{INTRODUCTION}

Electronic and smart health-care systems have changed the way we receive care and have improved quality and reduced cost [1]. In electronic health-care systems, many stakeholders collaborate with the aim to provide the right

\begin{tabular}{|l|l|}
\hline Access this article online \\
\hline DOI: 10.21928/undjst.v2n2y2018.pp24-31 & $\begin{array}{l}\text { E-ISSN: 2521-4217 } \\
\text { P-ISSN: 2521-4209 }\end{array}$ \\
\hline
\end{tabular}

Copyright (C) 2018 Mahmud, et al. This is an open access article distributed under the Creative Commons Attribution Non-Commercial No Derivatives License 4.0 (CC BY-NC-ND 4.0) care at the right time within the right cost. Achieving the aim is not without obstacles, and there are challenges many of which are yet to be addressed by researchers and system developers. Virtual health-care systems where patients receive care without face-to-face meetings are increasingly becoming the norm due to advances in communication technologies. We have previously suggested the use of virtual breeding environment (VBE) and virtual organization ( $\mathrm{VO}$ ) concepts for health care by Mahmud and Lu [2], and we have explained the benefits of using such concepts in providing virtual health care. In section 3.1, we introduce VBE and VO concepts briefly. One of the challenges of any virtual collaboration system

Corresponding author's e-mail: Hoger Mahmud, Department of Computer Science, College of Science and Technology, University of Human Development, Kurdistan Region, Iraq, Department of Computing Science, School of Computing and Engineering, University of Huddersfield, Huddersfield, UK 
is user verification and validation. To ensure the quality and integrity of health-care services provided through such virtual systems as well as preventing information falsification and identity thefts, user verification and validation are essential. Validation is necessary to ensure that the right provider with the right attribute as specified by the requester is selected and verification is necessary to ensure that the information provided is correct.

In this paper, we propose a framework that uses blockchain technology to verify and validate health-care providers in VBE-based health-care systems. In general, speaking blockchain records and stores transaction in a package called "block" and blocks are linked together in a distributed system. Blockchain technology is gaining interest to be used in various fields due to its flexibility in modifying the basic concept to be applied in various forms. Currently, well-known companies such as IBM, the Tierion/Philips partnership (Netherlands), Brontech (Australia), GEM (U.S.), and Guardtime (Europe) are applying and adapting the technology for their own particular needs [3]. For further clarification, we briefly introduce the blockchain concept in section 3.2.

A recent study by Deloitte has found that health-care providers are planning to use blockchain technologies in a wide scale as the technology gaining momentum both theoretically and practically. Zyskind et al. [4] suggested that blockchain technology can be a solution to the user identity verification problem that current authentication systems have a password, and dual-factor verification and validation mechanisms have not been successfully. The framework can also be used for record verification and validation which falls within user verification and validation issue. Statistics point to big health-care record keeping security issues, for example, in 2015 there were 112 million health-care record hacks [5]. Medical records are sensitive, and any alteration to its content may result in serious consequences. To ensure record integrity, blockchain can act as a distributed database that is secure and safeguard medical records against tempering [4].

The proposed framework does not present the technical aspects of implementing blockchain technology nor does it specify the blockchain mechanism to be used. As a first step, we have outlined the main information flow steps and have identified the required parties that should be members in a chain to verify and validate a health-care provider in VBE-based virtual health-care systems. We have also demonstrated the applicability of the framework in a simple but non-trivial virtual health-care scenario. This paper contributes toward the use of blockchain technology in health care in general and VBE-based health-care systems in specific for user verification and validation.

The rest of this paper is organized as follows: Section 2 provides some related research. In section 3 , we provide brief background information about VBE, VO, and blockchain as well as outlining and explaining the proposed framework. We demonstrate the use of the framework in section 4 and discuss the result in section 5 . We finally conclude in section 6 .

\section{RELATED WORK}

Blockchain concept was first introduced in 2008 [6], and later in 2009, the concept was implemented in creating the first cryptocurrency (Bitcoin) [7]. The technology is considered for use in health care and is already in use to provide a number of health-care services, for example, a system called "Prescrypt" is developed by Deloitte Netherlands in partnership with SNS Bank and Radboud3. The system enables patients to have full control over their data including allowing or revoking providers to access their data [8]. Some companies use blockchain in health care, for example, Gem (in collaboration with Philips Healthcare Blockchain Lab), PokitDok, Healthcoin, Hashed Health, and many others [9]. Other researchers have considered the use of blockchain technology for patient identification which allows a single person identification [10], and the use of blockchain in health care is considered by Alhadhrami et al. [1] for sharing health records between all relevant health-care stakeholders safely. As for data verification and validation in health care, the technology is used in various implemented and proposed systems, for example, the technology is used in developing a decentralized patient record database where data can be shared among many different parties with no concern for the integrity of the data [8]-[11]. Health bank (www.healthbank. coo) which is a Swiss company is planning to use blockchain to give full control of data usage to users through the use of the blockchain technology for transaction verification and validation. In a virtual healthcare setting the reputation of a care provider in terms of academic achievements and practical experience is one of the key selection attribute to provide a particular care. This is because care providers with high reputation presumed to provide better quality of care however the challenge here is how to verify and validate a reputation claim made by a care provider. Blockchain technology is proposed as a possible verification and validation technology for health-care provider reputations, for example, the authors of Sharples and Domingue [12] propose to use the technology in a system that can verify 
and validate educational records of health-care providers. The authors of Carboni [13] have developed a reputation model based on blockchain where customers can provide feedback after receiving a service from a provider and calculate the providers reputation based on feedbacks they receive. Gem Health Network launched by Gem a US startup uses blockchain technology to provide an infrastructure for health-care specialists to share information [14]. The technology is researched for fighting drug counterfeiting by Hyperledger in collaboration with Accenture, Cisco, Intel, IBM, Block Stream, and Bloomberg [15].

The technology is also considered and used in other fields, for example, it has been used in financial services such as online payment [16] and has been considered in other services such as smart contracts [17] and public services [18]. The Dutch Ministry of Agriculture is currently running a project called Blockchain for Agrifood that aims to explore the potential of blockchain technology in agriculture [19]. Blockchain is also used by the social enterprise "Provenance" (www.provenance.org) in the UK to record and validate certificates in agriculture supply chains. The technology is also used in music industry, for example, Startups such as Ujo or Peertraks propose to use the technology to manage music rights [20]. Our prosed use of the technology differs from all the above researches as we are the first (to the best of our knowledge) to suggest the use of blockchain technology for health-care provider verification and validation in VBE-based health-care systems.

\section{BACKGROUND AND FRAMEWORK DEFINITION}

In this section, we briefly introduce VBE, VO, and blockchain technology and we also define the proposed framework and explain its main steps.

\subsection{VBE and VO}

Internet and telecommunication technologies have paved the way for a new type of collaboration known as "virtual collaboration [21], [22]. The fact that virtual collaboration occurs between unknown participants has given rise to the challenge of collaboration management and regulation in a virtual world. To address the challenge, researchers have proposed VBE and VO [23], [24]. The concepts are researched for collaboration management and regulation in education, e-commerce, and teleworking [25], [26]. The framework proposed in this paper is specific to health-care services provided through systems which are developed based on VBE and VO. VO is a short-lived temporarily consortium where a number of parties collaborating and working together to provide a particular service. $\mathrm{VO}$ is described as "A loosely bound consortium of organizations that together address a specific demand that none of them can (at the given time) address alone and once the demand has been satisfied the VO might disband" [27]. VBE, on the other hand, is a permanent consortium of parties that provide the environment and support for $\mathrm{VO}$ creation and management. Participants in both VBE and VO can be human or machines or both, but they all have to collaborate through communication technologies [28].

\subsection{Blockchain}

Blockchain concept was developed from Bitcoin paper published by Nakamoto in 2008. It is a peer-to-peer network where all participants (peers) serve as a node and all nodes hold the same information (hash value in this case).

Blockchain uses cryptographic techniques to record transactions between peers in a peer-to-peer network and store the transaction in a digital ledger as a block. Blocks are linked together for validation and verification purposes. Each block is comprised of three main parts which are block headers, a hash value of the previous transaction, and Merkle root as illustrated in Fig. 1.

Each block contains a unique hash value that is the transaction recorded and distributed to all nodes in the chain after its creation and all have to agree before a change in the block can happen. The uniqueness of a hash value comes from the fact that any combination of data produces a unique hash value and this value changes if there is any alteration to the data; this mechanism ensures data validity. The use of cryptographic techniques in blockchain enhances the security of the data within a transaction which is an essential requirement of any health-care system. Blockchain uses the public key cryptographic technique to encrypt transactions, and it is visible to all participants in a blockchain; however, to decrypt

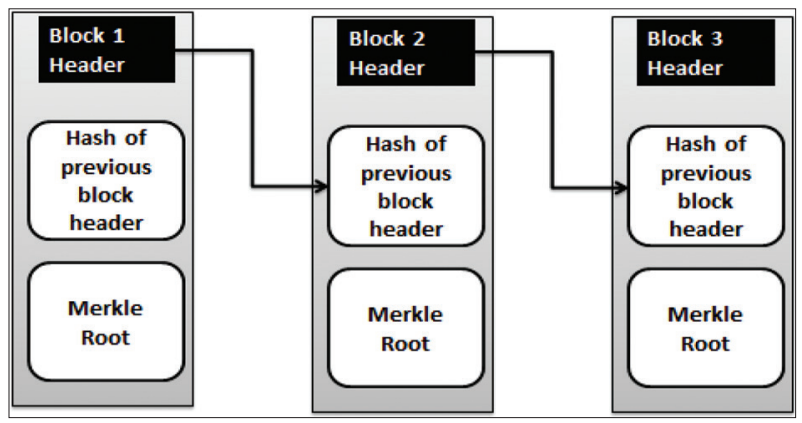

Fig. 1. Blocks linked in a chain.

UHD Journal of Science and Technology | May 2018 | Vol 2 | Issue 2 
Hoger Mahmud, et al.: A Blockchain-based Service Provider Validation and Verification Framework for Health-care Virtual Organization

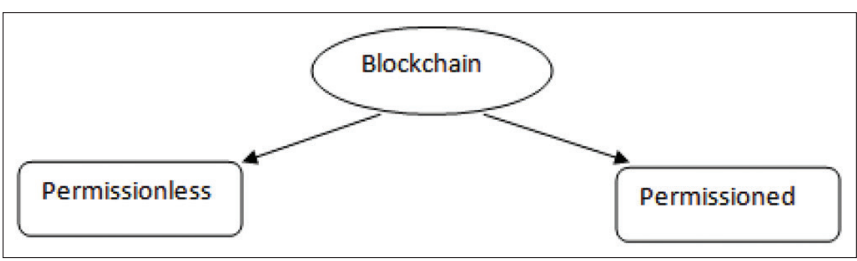

Fig. 2. Types of blockchain.

a transaction, a participant must have a private key which is not publically available [29]. In general, there are two types of blockchain which care for permissionless and permissioned blockchain. In a permissioned type of blockchain, a central authority controls all requests for change to transaction records or any other modification and the requester will have to go through access controls such as identity verification to access transactions [30]. On the other hand in permissionless blockchain, there is no central authority and requests can be made freely to change transaction records. Examining which type of blockchain is most suited for health-care virtual collaboration is beyond the scope of this paper as we only outline a framework without going into technical details; however, we think that it is an interesting topic to research. Fig. 2 illustrates the two types of blockchain.

\subsection{Framework Definition}

Here, we outline the proposed framework and provide more insights to each of the framework steps. Our proposed framework is conceptual rather than structural, and the purpose is to provide VBE-based health-care system developers with a step-by-step guide as to how to verify and validate health-care service providers and records using blockchain technology. For the framework to work, the following requirements should be fulfilled:

1. A virtual health-care system must use VBE and VO concept as a base for collaboration and organization of care provision which means that there must be a virtual environment where patients can send requests to and the environment creates a $\mathrm{VO}$ for the service requested after all requirements are fulfilled.

2. Service providers are recruited either within the VBE or from a global pool of virtual health-care providers after their credentials are verified and validated.

3. A blockchain is created between a number of VBEs, academic institutes, and health institutes where credentials of care providers are shared in blocks between all participants. Each blockchain participant has a job to do as follows:

a. VBEs: It provides information about health-care providers that have provided care within their environments for reputation verification and validation purposes. VBEs can also take part in health record verification and validation through sharing their records in the created blockchain.

b. Academic institutes: It provides information about the qualification that health-care providers claim to possess for credential verification and validation.

c. Health institutes: These provide information about the practices and experiences of health-care providers in real life situation and verify and validate the level of expertise and experience that providers claim to possess.

After all the above requirements are fulfilled, we suggest an eight-step framework which is illustrated in Fig. 3 to verify and validate providers and records as follows:

1. A health-care service request is triggered: This step serves as the trigger for the whole validation and verification process. In this step, a patient sends a request to a VBE for a virtual health-care service; for example, a patient would like to consult a doctor about a pain that he/she has developed in the neck after a minor car accident. The request can also be for a change of record that is held by a particular VBE, for example, a patient would like to make changes to the address registered in his/her record.

2. A health-care service accepted: VBEs cannot provide all types of care since health-care services are many and can change on a case bases; therefore, the VBE would have to check the details of the request to see if the requested service falls within their scope of work. If the request passes the check, then it is the job of the VBE to find the right health-care service provider after which a VO is created to provide the care. To do so, the VBE searches within its resources for the right service provider if not found the VBE would have to search the global pool for the right care provider.

3. After a provider is found, contacted, and accepted to offer the service, the VBE would have to verify and validate the credentials of the provider before final go-ahead for the service provision and VO creation. If the request is for changes in records held by the VBE, the credentials of the requester should be verified and validated before the change can be made.

4. VBE share the credentials: After step 3, the VBE would now have to share the record or the provider details with other participants using blockchain technology for verification and validation.

5. Blockchain-based verification and validation: When the information is shared, now each node in the chain would compare the information provided with the record held in blocks within their system for verification 
Hoger Mahmud, et al.: A Blockchain-based Service Provider Validation and Verification Framework for Health-care Virtual Organization

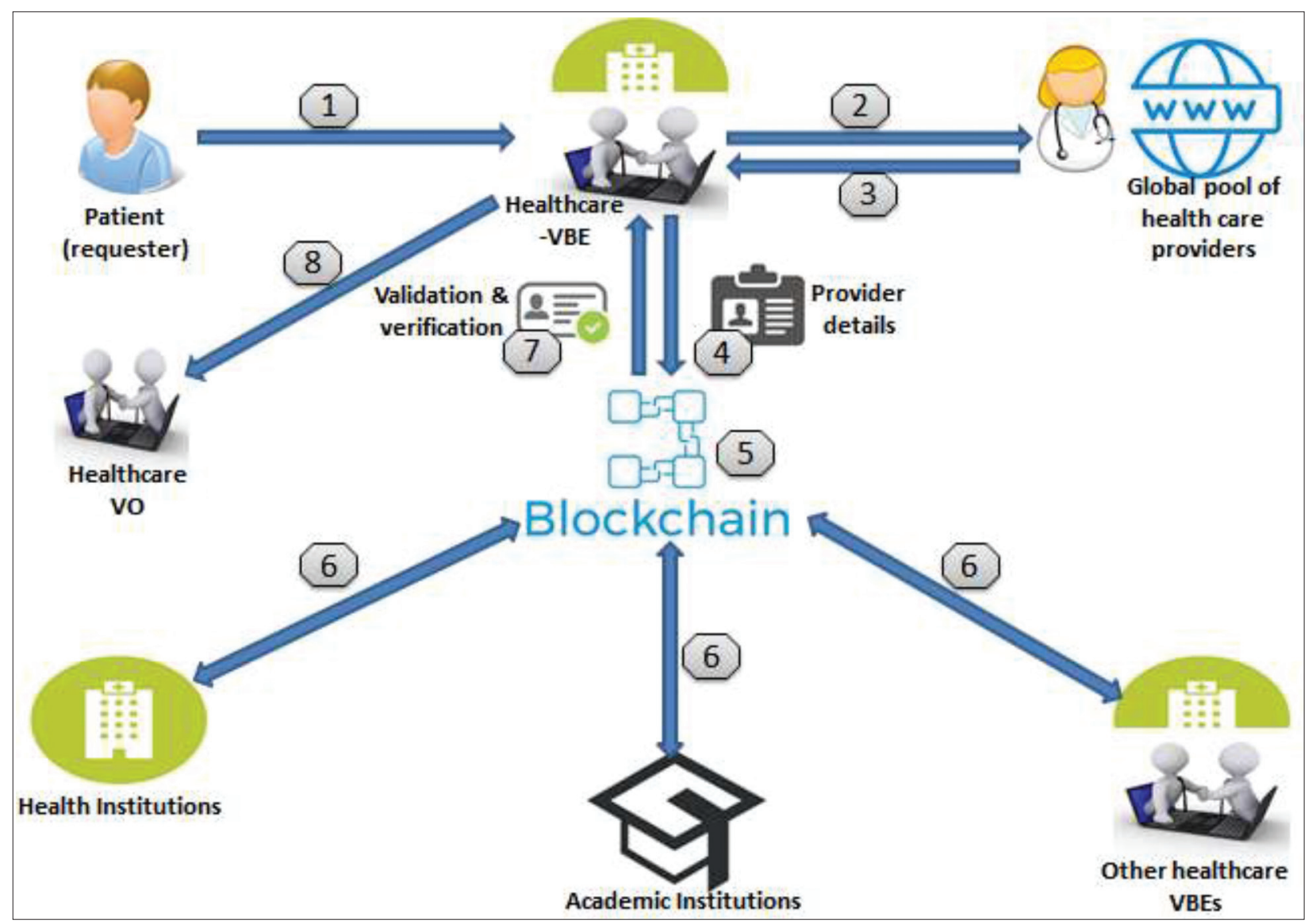

Fig. 3. The proposed framework.

and validation. The comparing process is done using consensus algorithms such as proof of work. In section 3.2 we have explained that blockchain is a peer-to-peer network and each peer in the network is a node that holds copies of transactions made in the network. When a new block is created, it is distributed to all nodes. The nodes will have the responsibility to validate the content of the block through comparing it with the block that is already held by the node.In blockchain an exact copy of a transaction (block) is held by all nodes in the chain, when a block is changed the request for change has to be broadcastand all nodes would have to approve the change to the block before a new block with the requested change is added to the network. In this case, if a service provider has provided false information, or if a record content is altered, it would be detected and rejected easily. This method of validation and verification is more robust than the insystem verification and validation since a record held in a system database can be hacked or altered, whereas in blockchain, it is impossible to alter data without all participant approval.

6. New block validation and creation: Sometimes, a request is send by a VBE where its content is new, for example, a care provider qualification needs to be verified and validated. In this case, the request would have to be compared with the records held by an academic institution, and once verified and validated, a new block would be created and added to the network. The step six is there for two purposes, the first is that participants would work as a peer in the network to provide verification and validation for blocks already created, and the second is to create new blocks and add it to the network as requests for information verification and validation comes into the network.

7. Request result: Once the result of the request is complete, it is sent back to the VBE, if the result is positive, the VBE would take steps to create a VO for the service otherwise new service provider has to be found, and the steps 2-7 have to be repeated or the whole process is stopped and the requester is informed of the reason.

8. VO creation: A VO is a short-lived entity created 
to provide a specific service, and once the goal is achieved, the VO is dismantled and the service ends. If the result of step 7 is positive, then a $\mathrm{VO}$ is created where both service requesters and service providers can communicate and collaborate. The process of VO creation mechanism is beyond the scope of this paper as we are currently researching on actively.

\section{CASE STUDY}

One of the requirements of a service requested is that the service has to be feasible virtually, i.e. the service requested has to be achievable through an online system. Healthcare services are complex with some requiring face-to-face meetings between care requesters and providers, and others can be achieved in a virtual system. One of the most common virtual heath-care service requests is for consultation. This where a patient would like to receive guidance about a particular medical needs or addresses a concern he/she may have. To show simply and effectively the contribution of the framework in verifying and validating care providers and records, we consider the scenario below:

Mr. Adam has recently been involved in a car accident and has developed a neck pain after the accident. Despite visiting a hospital a couple of times, the pain is still present and he would like to consult with a bone specialist that was not available in his local hospital. A VBE called "Virtual Hospital System" is introduced to him by a friend, and now he would like to contact the VBE for a service. He fills in the virtual care request form for a consultation service with a bone specialist. He specifies in his request that the bone specialist should have a good reputation and minimum 5 years of care provision experience. The specialist should be an EU graduate and speak very good English. It is now the job of the VBE to find the right specialist for Mr. Adam. To ensure the right specialist if put into contact with $\mathrm{Mr}$. Adam in a VO, the VBE uses the proposed framework and take the following steps:

1. The VBE searches though its database for a specialist that fulfills the requirements, but we assume that it fails to find one. The VBE then broadcasts the request and search for the right specialist in the global pool of care providers.

2. In the search process, the details of a specialist who lives in different countries than that of Mr. Adam match the requirements specified in the request form. The VBE contacts the specialist and offers to recruit him to provide the service and he accepts. In his profile, he claims that he is a UK-based university qualified with 7 years of experience in a German-based hospital. However, since the specialist is unknown to the VBE, the claims have to be verified and validated before the final go ahead.

3. The VBE create a block using the information provided by the specialist and broadcast it for verification and validation in the created blockchain. Now using blockchain mechanisms, the claims can easily be verified and validated by comparing the information in the block with those held by the network participants.

4. The result is sent back to the VBE and if positive put both Mr. Adam and the specialist into contact by creating a VO for them, and otherwise, the VBE withdraws the recruitment offer made to the specialist and search for another one or terminate the process.

The above scenario can simply demonstrate the applicability and the contribution of the framework in a clear manner; however, it must be said that the framework is conceptual and yet to be implemented for a real test which is something we still working on alongside other concepts to create the first VBE-based health-care system. The purpose of this paper is to share the principal concept and build on it in later works.

\section{DISCUSSION}

Ever since the Alma-Ata world leaders meeting that declared health care as a fundamental human right, many efforts and investments have been channeled through different healthcare systems around the world to ensure the delivery of this right. However, the main goal which was every human is entitle to receive quality care which is yet to be realized and this led to the World Health Organization to call for universal health-care coverage [31]. It is a known fact that most health-care systems are failing care receivers due to lack of stakeholder data safety, unacceptable quality of care, and limited care availability which all point to the need for change in health care. In the search for new ways to provide health care blockchain technology is seen by many researchers as a revolution with the potential to change the way heath care is provided currently [32], [33]. In this paper, we have outlined a framework that uses blockchain to address one of the most known issues in health care that can be provided virtually which is user verification and validation. Despite the invention of many techniques such as username and password authentication to ensure the identity and validity of the claims that are made by virtual care providers and verify their suitability to provide a requested care, the issue remains at large. The proposed framework is developed to 
contribute to the ongoing work to address the issue. The framework is simple and feasible as all technologies required to apply the framework are available. However, the framework is conceptual and required to be implemented and tested to show its full potential in contributing to the issue of data verification and validation in virtual health-care systems. The main contribution of this paper is the consideration of using blockchain in VBE-based health-care systems for service provider and records verification and validation as a concept, and the aim is to pave the way for further research and provide a basic validation and verification guide to system developers. As we have presented in this paper, blockchain technology is being considered for use in health care to address various issues in the field. However, despite the apparent theoretical applications of blockchain technologies in health care, the technology is yet to be applied fully due to its infancy and lack of technical implementation knowledge. One of the downsides of blockchain technology is the fact that operation costs are difficult to estimate as the computing power required to run it changes continuously as the number of hot nodes changes in the chain [5]. However, Blockchain technology has the potential to be used in health-care areas such as medicine authenticity identification and patient record sharing. Swan [8] identified a number of opportunities that blockchain technology can provide in health care such as:

1. Removal of third party between health-care providers and receivers as well as various health-care providers

2. Minimizing transaction costs as all transactions are transparent, direct, and happen real time

3. Ensuring the data shared between healthcare stakeholders is the last updated version as changes to stakeholder records are made real-time and updates are distributed to all nodes in the chain.

4. Creating one single and secure patient record access mechanism

\section{CONCLUSION}

Health-care provision is changing as different techniques are proposed to make health care more available and accessible with better quality and less cost. One of the techniques that are becoming familiar is receiving care through online without face-to-face meetings which is known as e-health or virtual health care. The technique has a number of challenges which are yet to be addressed fully, and one of which is record and service provider verification and validation. In this paper, we have outlined an eight-step framework that uses blockchain to address the issue in VBE-based health-care systems. The framework is conceptual and yet to be implemented, but we have demonstrated its applicability through applying it to a simple scenario that results in verifying and validating a care provider. This paper contributes toward tackling the challenge of verifying and validating users and records in health care and considers the use of blockchain for the first time in VBE-based healthcare systems. We plan to research further the possibility of implementing and testing the framework to uncover its full potential for virtual health-care systems.

\section{REFERENCES}

[1] Z. Alhadhrami, S. Alghfeli, M. Alghfeli, J. A. Abedlla and K. Shuaib. "Introducing blockchains for healthcare." Electrical and Computing Technologies and Applications (ICECTA), 2017 International Conference, pp. 1-4, 2017.

[2] H. Mahmud and J. Lu. "A generic vobe framework to manage home healthcare collaboration," Journal of Theoretical and Applied Information Technology, vol. 80, no. 2, p. 362, 2015.

[3] C. Stagnaro. "White Paper: Innovative Blockchain Uses in Health Care."

[4] G. Zyskind, O. Nathan and others. "Decentralizing privacy: Using blockchain to protect personal data." Security and Privacy Workshops (SPW), 2015 IEEE, pp. 180-184, 2015.

[5] C. P. Transaction. "Blockchain: Opportunities for Health Care." CP Transaction, 2016.

[6] S. Nakamoto, "Bitcoin: A peer-to-peer electronic cash system." CP Transaction, 2008.

[7] Z. Zheng, S. Xie, H. Dai, X. Chen and H. Wang. "An overview of blockchain technology: Architecture, consensus, and future trends.," Big Data (BigData Congress), 2017 IEEE International Congress on, pp. 557-564, 2017.

[8] M. Swan. "Blockchain: Blueprint for a new economy." California: O’Reilly Media, Inc, 2015.

[9] "How Blockchain Can Solve Real Problems in Healthcare." Available: https://www.linkedin.com/pulse/how-blockchain-cansolve-real-problems-healthcare-tamara-stclaire. [Jun. 5, 2018].

[10] L. A. Linn and M. B. Koo. "Blockchain for health data and its potential use in health it and health care related research." ONC/ NIST Use of Blockchain for Healthcare and Research Workshop. Gaithersburg, Maryland, United States: ONC/NIST, 2016.

[11] "The Blockchain for Healthcare: Gem Launches Gem Health Network With Philips Blockchain Lab." Available: https://www. bitcoinmagazine.com/articles/the-blockchain-for-heathcaregem-launches-gem-health-network-with-philips-blockchainlab-1461674938. [May 02, 2018].

[12] M. Sharples and J. Domingue. "The blockchain and kudos: A distributed system for educational record, reputation and reward." European Conference on Technology Enhanced Learning, pp. 490-496, 2016.

[13] D. Carboni. "Feedback based Reputation on top of the Bitcoin Blockchain.," arXiv preprint arXiv:1502.01504, 2015.

[14] "Gemhealth." Available: https://www.bitcoinmagazine.com/articles/ the-blockchain-for-heathcare-gem-launches-gem-health-networkwith-philips-blockchain-lab-1461674938/. [Apr. 2, 2018].

[15] "Applying Blockchain Technology To Medicine Traceabilit." 
Available: https://www.securingindustry.com/pharmaceuticals/ applying-blockchain-technology-to-medicine-traceability/s40/ a2766/\#.W1hTsNIzblU.[Last accessed on 2018 Mar 27].

[16] G. Peters, E. Panayi and A. Chapelle. "Trends in crypto-currencies and blockchain technologies: A monetary theory and regulation perspective." Journal of Financial Perspectives, pp. 38-69, 2015.

[17] A. Kosba, A. Miller, E. Shi, Z. Wen and C. Papamanthou. "Hawk: The blockchain model of cryptography and privacy-preserving smart contracts." 2016 IEEE symposium on security and privacy (SP), pp. 839-858, 2016

[18] B. W. Akins, J. L. Chapman and J. M. Gordon. "A whole new world: Income tax considerations of the Bitcoin economy." Pittsburgh Tax Review, vol. 12, p. 25, 2014

[19] L. Ge, C. Brewster, J. Spek, A. Smeenk, J. Top, F. van Diepen, B. Klaase, C. Graumans and M. de R. de Wildt. "Blockchain for Agriculture and Food." Wageningen Economic Research, p. 112, 2017.

[20] M. Mettler. "Blockchain technology in healthcare: The revolution starts here." e-Health Networking, Applications and Services (Healthcom), 2016 IEEE $18^{\text {th }}$ International Conference on, pp. 1-3, 2016

[21] R. P. Biuk-Aghai and S. Simoff. "Patterns of virtual collaboration in online collaboration systems." Proceedings of the IASTED International Conference on Knowledge Sharing and Collaborative Engineering, St. Thomas, USVI, pp. 22-24, Nov, 2004.

[22] L. Wainfan and P. K. Davis. "challenges In Virtual Collaboration: Videoconferencing, Audioconferencing, and Computer-Mediated Communications". Rand Corporation, 2004.

[23] C. Zirpins and W. Emmerich. "Virtual organisation by service virtualisation: Conceptual model and e-science application." Research Notes RN/07/07, University College London, Dept. of Computer Science, 2007.
[24] E. Ermilova and H. Afsarmanesh. "Modeling and management of profiles and competencies in VBEs." Journal of Intelligent Manufacturing, vol. 18, no. 5, pp. 561-586, 2007.

[25] P. R. Messinger, E. Stroulia and K. Lyons. "A typology of virtual worlds: Historical overview and future directions." Journal For Virtual Worlds Research, vol. 1, no. 1, 2008.

[26] J. M. Balkin and B. S. Noveck. "State of Play: Law, Games, and Virtual Worlds: Law, Games, and Virtual Worlds (Ex Machina: Law, Technology, and Society)". New York: NYU Press, 2006.

[27] S. Reiff-Marganiec and N. J. Rajper. "Modelling virtual organisations: Structure and reconfigurations." Adaptation and Value Creating Collaborative Networks, pp. 297-305, 2014.

[28] H. Afsarmanesh and L. M. Camarinha-Matos. "A framework for management of virtual organization breeding environments." Collaborative Networks and Their Breeding Environments, pp. 3548, 2005.

[29] A. Salomaa. "Public-key Cryptography". New York: Springer Science and Business Media, 2013.

[30] A. Collomb and K. Sok. "Blockchain/distributed ledger technology (DLT): What impact on the financial sector?" Communications and Strategies, no. 103, p. 93, 2016.

[31] B. M. Till, A. W. Peters, S. Afshar and J. G. Meara. "From blockchain technology to global health equity: can cryptocurrencies finance universal health coverage?" BMJ global health, vol. 2, no. 4, p. e000570, 2017.

[32] I. C. Ellul. "Blockchain and Healthcare: Will there be Offspring?" Sweden: Palestinian, 2017.

[33] D. Randall, P. Goel and R. Abujamra, "Blockchain applications and use cases in health information technology." Journal of Health and Medical Informatics, vol. 8, no. 3, 2017. 